\title{
Achondrogenesis Type II, Abnormalities of Extracellular Matrix
}

\author{
WILLIAM A. HORTON, MIRTA A. MACHADO, JEAN W. CHOU, AND DIANNA CAMPBELL
}

Department of Pediatrics, University of Texas Medical School, Houston, Texas 77225

\begin{abstract}
Immune and lectin histochemical and microchemical methods were employed to study growth cartilage from seven cases of achondrogenesis type II (LangerSaldino). The normal architecture of the epiphyseal and growth plate cartilage was replaced by a morphologically heterogeneous tissue. Some areas were comprised of vascular canals surrounded by extensive fibrous tissue and enlarged cells that had the appearance and histochemical characteristics of hypertrophic chondrocytes. Other areas contained a mixture of cells ranging from small to the enlarged chondrocytes. The extracellular matrix in the latter areas was more abundant and had characteristics of both precartilage mesenchymal matrix and typical cartilage matrix; it contained types I and II collagen, cartilage proteoglycan, fibronectin, and peanut agglutinin binding glycoconjugate(s). Peptide mapping of cyanogen bromide cartilage collagen peptides revealed the presence of types I and II collagen. These observations could be explained by a defect in the biosynthesis of type II collagen or in chondrocyte differentiation. (Pediatr Res 22:324-329, 1987)
\end{abstract}

\section{Abbreviations}

PG, proteoglycan

C6S PG, chondroitin-6-sulfate PG

KS PG, keratan sulfate PG

PNA, peanut agglutinin

UEA, ulex europaeus agglutinin

GAG PS, glycosaminoglycan PG

$\mathrm{CI}_{\text {femur }}$, femoral cylindrical index

BSA, bovine serum albumin

PBS, phosphate-buffered saline

Achondrogenesis type II (Langer-Saldino) is a rare autosomal recessive bone dysplasia characterized by profound shortening of the limbs, distinctive radiographic changes, and lethality in the newborn period (1-3). Characteristic histologic abnormalities of growth plate cartilage have been described by several investigators (3-5) which include an increased number of vascular canals, increased amount of fibrous tissue, and most notably replacement of the normal architecture of growth cartilage by poorly organized masses of hypertrophic-like chondrocytes and sparse extracellular matrix. In addition, we and others have reported immunohistochemical and microchemical studies indicating that

Received October 28, 1986; accepted April 14, 1987

Reprint requests William A. Horton M.D., Department of Pediatrics, University of Texas Health Science Center, P.O. Box 20708, Houston, TX 77225.

Supported by Research Grants HD 20691 and AG 04661 from the National Institutes of Health, March of Dimes Birth Defects Foundation (Clinical Research Grant 6-363), and Shriners of North America (15953). the predominant collagen of the growth cartilage in this disorder is type I rather than type II collagen as is normally found (6-9). Although these various changes presumably reflect the basic defect in achondrogenesis type II, the nature of the defect and how it disrupts linear bone growth are not known. To explore these issues, we have studied epiphyseal and growth plate cartilage from seven cases of achondrogenesis type II by a combination of morphologic, immune, and lectin histochemical and microchemical methods designed to determine the distribution and content of skeletal collagens, proteoglycans, and other extracellular matrix macromolecules in the tissue. Our results suggest that achondrogenesis type II may be a disorder of type II collagen biosynthesis or of chondrocyte differentiation.

\section{METHODS}

Immune reagents and standards. The antibodies directed against type I collagen were monoclonal antibodies provided by Dr. Steffen Gay, University of Alabama, Birmingham, whereas the antibodies to type II collagen were affinity purified polyclonal antibodies. The monoclonal antibodies to GAG PG determinants C6S PG and KS PG were gifts from Dr. Bruce Caterson, University of West Virginia, Morgantown; they have been previously designated $5 / 6 / 3 \mathrm{~B} 3$ and $1 / 21 / 5-\mathrm{D} 4$, respectively. The preparation and characterization of these antibodies have been reported previously $(10-12)$. The polyclonal antibody to fibronectin was purchased from Miles Laboratories. The biotinylated lectins and $\mathrm{ABC}$ kits were purchased from Vector Laboratories (Burlingame, CA). Rabbit anti-mouse and swine anti-rabbit immunoglobulins conjugated to horseradish peroxidase were obtained from Accurate Scientific (Westbury, NY). The standards for type I collagen, $\alpha 1(\mathrm{I})$ and type II collagen, $\alpha$ 1(II) were prepared by standard techniques from human skin and costochondral cartilage respectively.

Tissue sources and preparation. Growth cartilage specimens were obtained from multiple sites, including costochondral junction, proximal and distal femur, and iliac crest, from seven fetuses/newborn infants who exhibited radiographic abnormalities typical of achondrogenesis type II (Langer-Saldino). $\mathrm{Cl}_{\mathrm{femur}}$ were determined to subdivide them according to the scheme of Whitley and Gorlin (13). One had a $\mathrm{CI}_{\text {femur }}$ of 2.5 which placed her in the severely affected group (Whitley and Gorlin type II), one had a $\mathrm{CI}_{\text {femur }}$ of 7.2 assigning him to the mild group (Whitley and Gorlin type IV), and the other had $\mathrm{CI}_{\mathrm{femur}}$ ranging from 3.7 to 4.4 placing them in the moderately severe group (Whitley and Gorlin type III). They ranged in gestational age from 18 to 40 wk. Autopsy specimens from 10 comparable-aged fetuses and newborn infants not having bone dysplasias were used as controls. The specimens contained growth cartilage tissue that normally is occupied by epiphyseal and growth plate cartilage and subchondral bone. Several specimens were referred from other institutions. Some were sent by air express in transport medium. Others were divided into representative portions of which some were fixed in $10 \%$ buffered formalin and shipped in buffer and 
some were frozen and transported on dry ice. Tissues obtained locally were lixed overnight at $4^{\circ}$ ( in buffered $10 \%$ formalin or in $4 \%$ paraformaldehyde. $1 \%$ glutaraldehyde, and $0.07 \%$ ruthenium hexamine trichloride in sodium cacodylate buffer, pH 7.4 (11, 14). They were dehydrated through graded ethanol and infiltrated and embedded in Spurr epoxy resin by standard techniques. In addition. some specimens were cmbedded in glycol methacrylate and examined by routine histochemistry (6).

Six of the specimens were studied hiochemically. The loose connective lissuc if present was carefully removed from the unfixed epiphyseal cartilage and cross-sectional dises approximately $3(0)-40(0) \mu$ thick were cut on a McIlwain tissue slicer These were cut no closer than $300 \mu$ from the cartilage bone interface to assure that no bone was included in the disc. The dises were stored at -8()$^{\circ}(\mathrm{C}$

Immumohistorhemical staining. Sections $1.5-\mu$ thick of undecalcified cartilage and bone were placed on acid cleaned subbed slides for all studies. Spurr was removed from the sections in at 1:1:1 solution of $1 \%$ polassium hydroxide in methanolacetone:benerene for $15 \mathrm{~min}(15)$. After neutralization in a $1 \%$ acetic acid solution in methanol for 1 min. the sections were rinsed in absolute methanol and placed in $5 \% \mathrm{H}_{2} \mathrm{O}$, in methanol for $15 \mathrm{~min}$ to remove endogenous peroxidase activity. The sections were then hodrated through graded methanol to PBS. pH 7.2.

The immunohistochemical staining methods have been previously described $(6,11)$. To demonstrate the collagens and fibronectin, the sections were pretreated with $0.25 \mathrm{mg} / \mathrm{ml}$ Protease XIV (Sigma (hemical (o.. St. I.ouis. MO) in PBS for 10 min, rinsed in PBS, and treated with $5 \%$ BSA for 20 min. The sections were drained and incubated in primary antibody: $1 / 15$ dilution in $2 \%$ BSA for 18 hat $4^{\circ}$ ( for type I collagen. 1/40) dilution in $2 \%$ BSA for 2 h at $37^{\circ}$ ( for type II collagen, and 1 / 175 dilution in $2 \%$ BSA for 2 h at $37^{\circ}$ ( for fibronectin. The sections were then incubated with rabbit anti-mouse IgG conjugated with horseradish peroxidase for 2 h at $37^{\circ}$ ( for type I collagen and for $1 \mathrm{~h}$ at $37^{\circ}$ C with swine anti-rabbit conjugated with horseradish peroxidase for type II collagen and fibronectin. For control purposes, nonimmune rabbit and mouse sera were employed for the primary incubations. Horseradish peroxidase activity was demonstrated by incubation in $0.10 \% 3.3^{\prime}$-diaminobenzidine tetrahydrochloride (Polysciences Inc.. Warrington. $P A)$ and (0.17\% hydrogen peroxide. The sections were mounted in permount and photographed with a Ziess Photomicroseope III equiped with Nomarsky high-contrast optics.

Io demonstrate the (iAC; PC determinants the avidin-biotinperoxidase system ( $\triangle B C$. Vector Laboratories. $(A)$ was used (16). The sections to be stained for C COS PC $;$ were pretreated in chondroitinase $\triangle B C^{\circ}$ (Sigma (hemical) for 30 min at $37^{\circ} C^{\circ}$ in Tris Buffer, pH 7.6. (hondroitinase digestion was not needed to demonstrate KS P(j. The sections were covered with horse serum for $20 \mathrm{~min}$ and incubated in a $1 / 200$ dilution of the primary antibody in $2 \%$ BSA for $30 \mathrm{~min}$ at room temperature. Subsequently, they were placed in biotinylated anti-mouse $\operatorname{Ig}(i$ for 30 min. followed by a solution of avidin DH and biotinylated horseradish peroxide for $45 \mathrm{~min}$. Peroxidase activity was demonstrated as above. Primary incubation in nonimmune mouse serum was used for control specimens.

lectin histochemistrl. The binding pattern of two lectins was examined: UFA which binds to fucose containing glycoconjugates and PNA which binds to gatactose containing glycoconjugates $(17,18)$. For staining the Spurr resin was removed from the sections as described above, and they were hydrated in PBS. They were then incubated for $30 \mathrm{~min}$ at room temperature in biotinylated lectins diluted 1:400) in PBS. washed. and incubated in Vectastain $A B C$ reagent (Vector Laboratories). Horseradish peroxidase activity was demonstrated as above. Inhibitory sugars (fucose for (JEA and galactose for PNA) were added to the primary incubation of adjacent sections for control purposes.

collagen peptide mapping. After thawing the cartilage discs. cyanogen bromide collagen peptides were prepared from aach dise and chromatographed as previousl described (\$). Briefly. the dises were placed overnight in $4.0 \mathrm{M}$ guatnidine $13(1$ containing protease inhibitors. Afier vacuum filtration. rinsing. and drying. they were dissolved in 7()$^{\circ}$; formic acid to which a 10(0) fold excess of evanogen bromide was added. The digestion wats carried out at $30^{\circ}$ ( for $t h$. after which the digest was diluted with distilled water and lyophilized. The preparations were dissolved in $50 \mathrm{mM}$ Tris pH 7.4 containing 2.0 $\mathrm{M}$ guanidine $\mathrm{HCl}$ filtered and aliquots corresponding to $25 \mu \mathrm{g}$ of collagen peptides were chromatographed over two tandomly arranged $7.5 \times 30()$ mm TSK gel filtration HPL. ( columns (Bio-Sil ISK 250). Biorad). The flow rate was $0.6 \mathrm{ml} / \mathrm{mm}$, and the chromategraphy was monitored at $225 \mathrm{~nm}$. The data were analyed and stored and chromatograms compared to those from similarly prepared collagen standards as well as collagen from normal epiphyseat cartilage on a Velson analytical system model 4416.

\section{RISTLIS}

All specimens. including those sent from other institutions. laken from fetuses of different gestational ages. procured from cases of different severity and obtained from different anatomic sites. showed the same general histologic appearance. There was no distinction between epiphyseal and growth plate cartilige and no organized growth plate. Cartilage canals were greater in number and larger than seen in age matched control specimens. They were irregularly distributed and comprised of vascular tissue and extensive perivascular fibrous connective tissue (Fig. 1). Two patterns of chondrocyte organization were observed. The first. designated hypertrophic (Fig. 2), was characterised by cells lying within large elongated lacunae that were separated by thin matrix septa. These lacunae tended to be arranged radially around the cartilage canals such that the long axes of the lacunac were perpendicular to the canals. The chondrocytes appeared withdrawn from the lacunar walls in the tissues fixed in formalin alone. They appeared to occupy a larger proportion of the lacunae in tissues to which ruthenium hexamine trichloride, an agent thought to prevent chondrocyte shrinkage. was added during fixation. However. in both instances granular material occupied the apparent space between the cell borders and the lacunar walls. The appearance of these cells resembled that of hypertrophic and degenerative chondrocyes from the growth plate in control specimens. In other areas designated mixed trig. 3). irregularly distributed chondrocytes varied in size from the

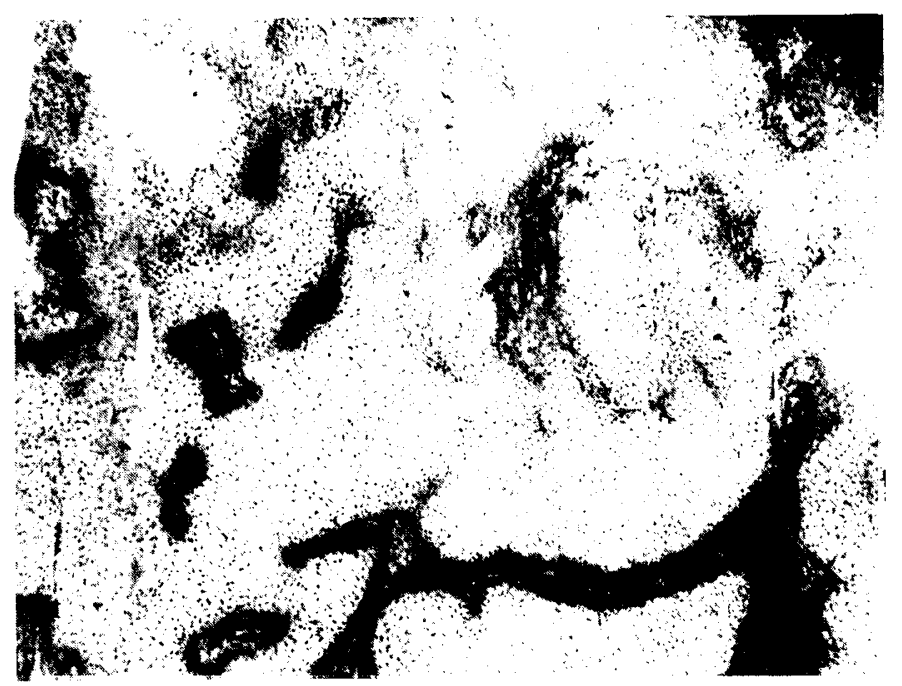

Fig. 1. Growth cartilage from a $36-w k$ fetus stained with methenamine silver to demonstrate general architecture. I)arkly stained areas indicate cartilage canals. (ilycol methacrulate section, original magnitication, $\times 50$. 

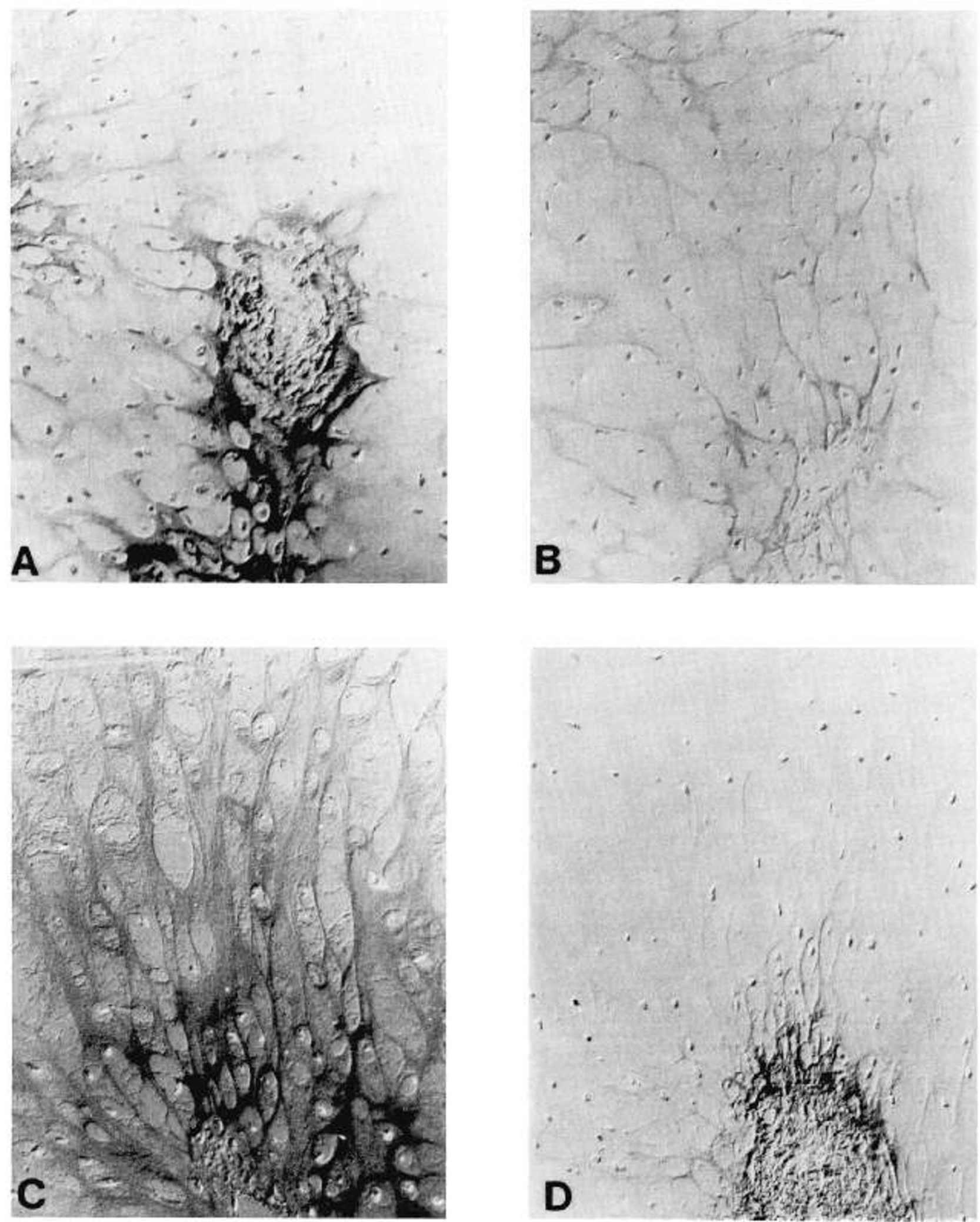

Fig. 2. Region of growth cartilage from a 16-wk fetus exhibiting the hypertrophic cartilage morphologic appearance stained to demonstrate: $A$, type I collagen; $B$, type II collagen; $C, \mathrm{C} 6 \mathrm{~S}$ PG; and $D$, PNA binding. The perivascular fibrous tissues stain intensely for type I collagen, C6S PG, and PNA and weakly for type II collagen. The matrix septa that separate the enlarged chondrocytes stain weakly for both type I and II collagen and moderately for C6S PG, but not for PNA. Note that the material located between the cell borders and lacunar walls stains for C6S PG. Original magnification, $\times 160$.

enlarged cells described above to small round cells within small lacunae. The extracellular matrix was more abundant in these areas, but was less than in controls. Poorly staining areas in which the matrix appeared very fibrous were scattered through- out these regions. The areas of hypertrophic and mixed cartilage were randomly intermixed and not well demarcated from one another. There was no correlation between the relative proportion of morphologic patterns and the radiographic severity, al- 

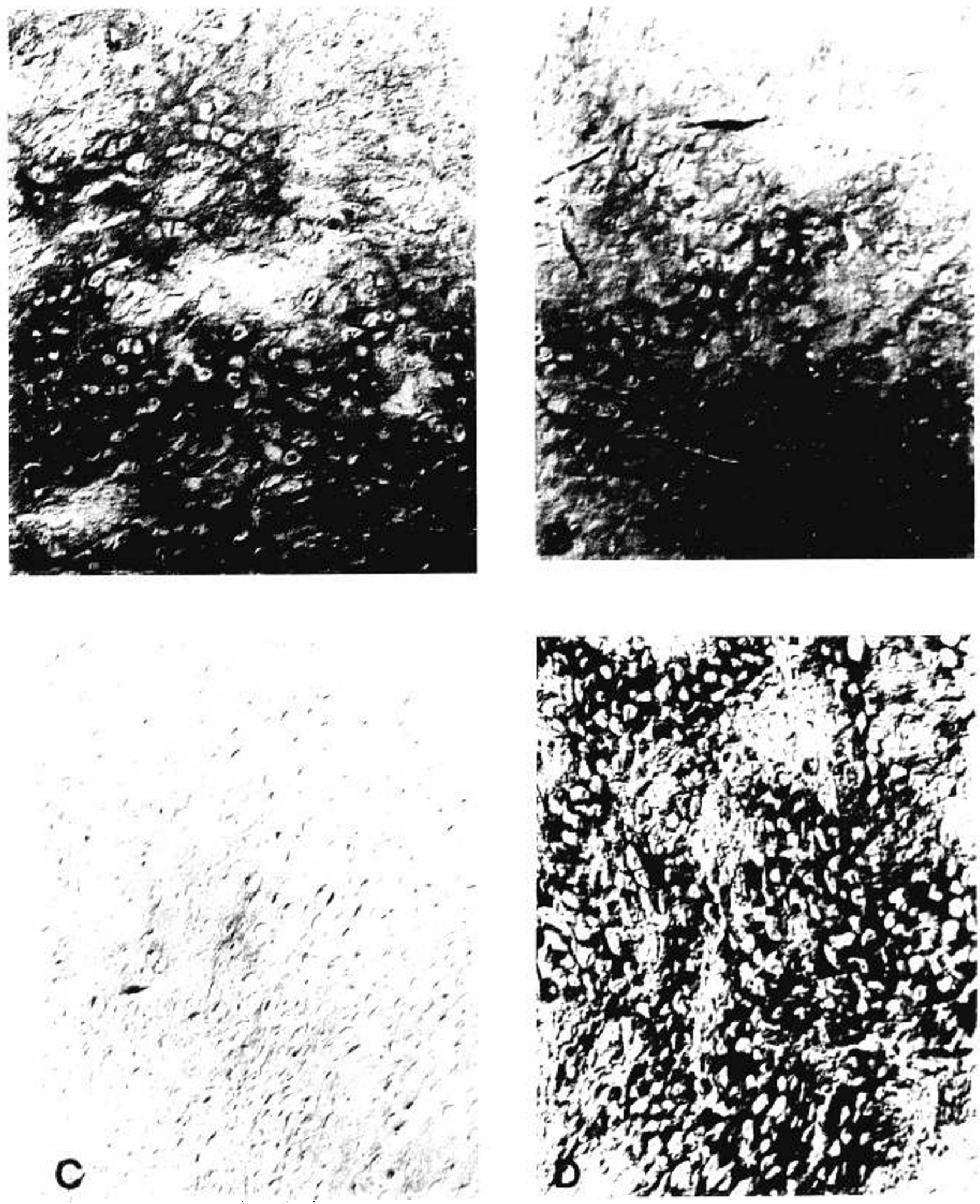

Fig. 3. Region of growth cartilage from a 16-wk fetus exhibiting the mixed cartilage morphologic appearance stained to demonstrate: 1 , type I collagen; $B$, type If collagen; $(, \operatorname{CoS} P(j ;$ and $D$, PNA binding. Note that the matrix is more abundant and stains for all four determinants. Original magnification, $\times 160$

though the matrix tended to be more abundant in the fetus exhibiting the mild changes $\left(\mathrm{CI}_{\mathrm{fermur}}=7.3\right)$.

In the hypertrophic cartilage, the perivascular fibrous tissue of the cartilage canals stained intensely for type I collagen, fibronectin and for the two lectins (Fig. $2 A$ and $D$ ). The staining pattern was similar for all four determinants and the staining intensity was greatest at the borders of the canals and the cartilage. Staining for these determinants extended a short distance into the cartilage matrix septa between the hypertrophic chondrocytes. There was weak staining for type II collagen near the cartilage canals and within the matrix septa (Fig. 2B). The distributions of C6S PG and KS PG were essentially the same. 
Both localized to the border region, and there was also moderately intense immunostaining of the matrix septa throughout these regions as well as the granular intralacunar material for both determinants (Fig. 2C).

The matrix of the mixed regions stained variably for types I and II collagen, fibronectin, both PG determinants and both lectins (Fig. $3 A-D$ ). In contrast only type II collagen and the GAG PG determinants were demonstrated in control cartilage matrix.

Cyanogen bromide peptides from epiphyseal cartilage collagens were analysed in six cases (Fig. 4). The elution profile was consistent with type II collagen, $\alpha$ 1(II), in one patient who was moderately severely affected $\left(\mathrm{CI}_{\text {femur }}=3.9\right)$. This patient had a gestational age of $20 \mathrm{wk}$. In the other five patients, including the mildly affected fetus $\left(\mathrm{CI}_{\mathrm{femur}}=7.3\right)$ and a fetus of $16 \mathrm{wk}$ gestation, the profiles differed from $\alpha 1$ (II) and contained marker peptides for type I collagen, $\alpha 1(\mathrm{I})$.

\section{DISCUSSION}

The nomenclature for achondrogenesis has become very confusing. Until recently, it was accepted that there were two classic types: Parenti-Fraccaro (type I) and Langer-Saldino (type II).

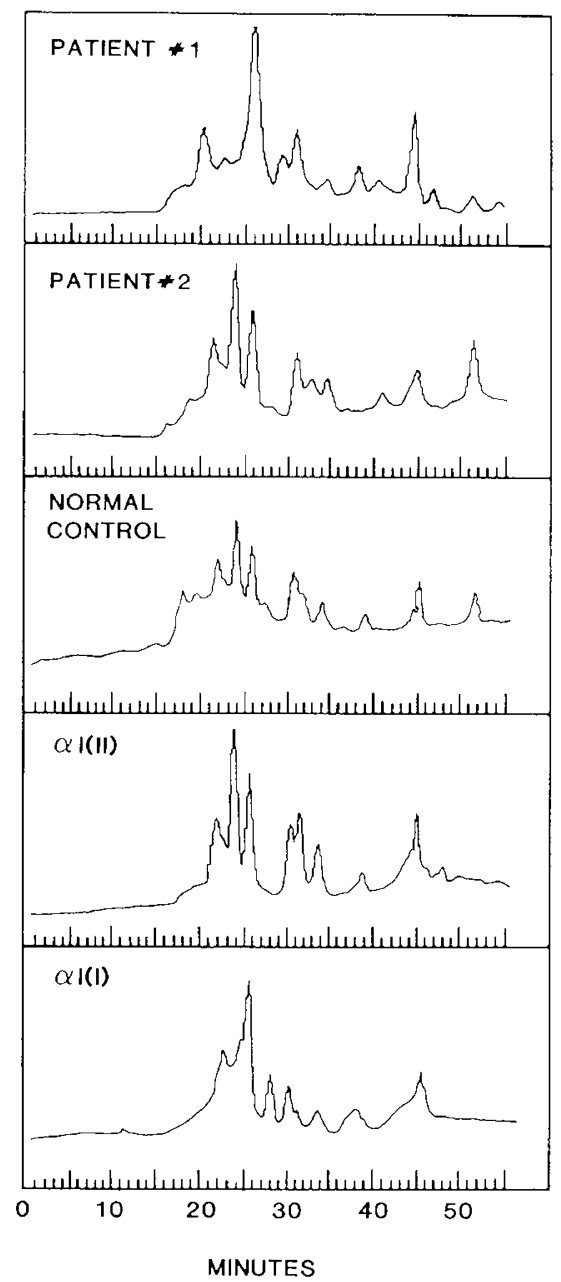

Fig. 4. Elution profiles for cyanogen bromide peptides from collagens prepared from growth cartilage from two patients compared to peptides from a similar preparation from normal control cartilage and from standards for types I and II collagen. Absorbance is plotted against time in minutes. The profile for patient 1 (representative of five patients) differs substantially from the profile for type II collagen and contains marker peptides for alpha 1(I). The profile for patient 2 is the same as that for the normal control cartilage and for the type II collagen standard.
This classification is employed in the International Nomenclature of Constitutional Diseases of Bone (19). However, Whitley and Gorlin (13) have suggested that type II be subdivided into genetically distinct severe, moderately severe and mild forms which they designated types II, III, and IV with type IV referring to hypochondrogenesis. Borochowitz et al. (20) disputed this view suggesting that there is a continuous spectrum of severity with typical achondrogenesis type II at one end and hypochondrogenesis at the other. Our results which were based on cases ranging from severe (Whitley and Gorlin type II) to mild (Whitley and Gorlin type IV, hypochondrogenesis), showed no substantial differences by the morphologic, histochemical, and microchemical methods employed. These findings do not necessarily refute the existence of genetically distinct forms of the disorder, but do suggest that if they exist they share a common pathogenetic mechanism.

Our results demonstrated that the normal architecture of growth cartilage is completely disrupted. As others have shown (3-5), much of the cartilage was highly vascularized and comprised of enlarged chondrocytes dispersed within sparse matrix. However, other less characteristic areas were observed that more closely resembled normal cartilage. The microchemical results provided evidence for the presence of both types I and II collagen in the growth cartilages. It could be argued that the finding of type II collagen in a cartilage sample from one patient and of type I collagen in cartilage samples from others supports the heterogeneity hypothesis discussed above. However, both types of collagen were demonstrated immunohistochemically in all the cases, and the discrepancy more likely reflects sampling differences, ie, type I collagen more prominent in hypertrophic regions where there was more vascular tissue and type II collagen in the mixed areas. Indeed, in an investigation of a bovine model of achondrogenesis type II, we have sampled different sites within the same growth cartilage and shown that the amount of type I collagen varies (21).

Since limb growth is so disturbed in this disorder, it seems reasonable to consider our observations in the context of normal limb development (22). This process begins with the outgrowth of limb buds comprised of mesenchymal tissue covered by a layer of ectoderm. As regionalization of the mesenchyme occurs, skeletal primordia can be identified as condensations of cells, so called precartilage condensations. The matrix associated with these condensations contains types I and III collagen and fibronectin $(18,23-26)$ as well as a galactose-rich substance to which PNA binds. Indeed, the latter has been used as a marker for this stage of chondrogenesis. Shortly after condensation, the mesenchymal cells differentiate into chondrocytes. This is associated with the synthesis of type II collagen and cartilage PG, the dispersement of cells within a cartilage matrix, the cessation of synthesis of types I and III collagen and fibronectin and the loss of binding of PNA (24-28). Primary ossification centers are formed in the diaphyses of the bones-to-be by vascular invasion of the cartilage anlagen. As these centers spread to near the ends of the bones where formal growth plates develop, the chondrocytes adjacent to the ossification front further differentiate into hypertrophic chondrocytes which subsequently degenerate and die as the ossification front reaches them. The differentiation to hypertrophic chondrocytes is accompanied by the renewed synthesis of type I collagen and fibronectin, the synthesis of a collagen specific for hypertrophic chondrocytes (type X collagen) and reduced synthesis of type II collagen and perhaps cartilage PG (29-34). A similar differentiation into hypertrophic chondrocytes occurs at secondary ossification centers.

The phenotype of the cells involved in limb development thus changes dramatically during the normal process; and this is reflected by the extracellular matrix macromolecules produced by the cells at different stages of differentiation as well as by their morphologic appearance. With the exception of the growth plate and secondary ossification centers, the overwhelming majority of cells occupying the cartilagenous portion of the normal fetal 
and newborn bone display the phenotype of the typical chondrocyte: and it is the expression of this phenotype that is most disturbed in achondrogenesis type II. Fyre of al. (9) have proposed that the basic defect in the disorder disrupts the biosynthesis of type II collagen, the major structural protein of cartilage (9). They eite the presence in the matrix of other elements of typical cartilage matrix, including $1 \propto x, 2 \propto, 3 \alpha$ collagen, type IX collagen, and cartilage proteoglycan to support this contention and suggest that the type I collagen is synthesized as a replacement for the missing type II collagen. Our findings support this view except that the detection of type II collagen in some instances would imply that the synthetic failure is incomplete. Perhaps a low level of synthesis of type Il collagen is possible at least in some cells within the growth cartilage.

Another hypothesis to explain the findings is that the maintenance of the typical chondrocyte phenotype is disturbed. It is well known that the typieal chondrocyte phenotype is unstable in vitro; cells tend to dedifferentiate into more primitive mesenchymal-like cells or further differentiate into hypertrophic chondrocytes (35-38). If such phenotypic instability were to occur in growth cartilage, one would expect to find a mixture of eell types ranging from precartilage mesenchymal cells to hypertrophic chondrocyles and an extracellular matrix containing macromolecules indicative of the different stages of chondrocyte differentiation. This is what was observed. Further speculation is not warranted. but it should be pointed out that the two hypotheses are not mutually exclusive. For example, it seems likely that type Il collagen is important in maintaining the typical chondrocyte phenotype and that a deficiency of this molecule might lead to phenotypic instability.

Thus a number of abnormalities in the composition of growth cartilage extracellular matrix have been demonstrated in achondrogenesis type II. Whether they are secondary to deficient biosynthesis of type II collagen or reflect an abnormality of chondrocyte differentiation or some other pathogenetic process remains to be determined.

\section{RFFHRIVIC}

1. Ianger 10) Spramger IW. (ircinacher I. Herdman R( 1969 thanatophoric dwadism. a condition confused with achondeoplasia in the neonate. with bricf comments on achondrogencsis and homorygous achondroplasia. Ra(jiology $92: 285.294$

2. Saldino RM 1971 I ethal short-limbed dwartism, achondrogenesis and thanatophoric dwarlism. AJR $112: 185.197$

3. Rimoin 1)1 1075 The chondrodystrophes Ad 11 um (jenet $5: 1 \ldots 118$

4. Yang S-S. Brough AJ. Ciarewal (iS. Sernstein J 1974 1 wo types of heritable lethal achendrogenesis. J Pediatr $85: 796-80)$

5. Sillence DO). Horton WA. Rimoin [)I, 1979 Morphologic studies in the skeletal dvsplasiass Am \& Pathol $96: 8 ! 1-870$

6. Horton WA 1984 Historhemistry a valuable tool in connective tissue research. (olligen Rol Res 4:231-237

7. Horton WA. Machado MA. (hou JW. Gyre DR 3985 Ahnormal cartilage collagens in achondrogenesis. Pediatr Res 19:248(abstr

8. Horton WA (hou JW Machado MA lo8s cartilage collagen analysis in the chondrodvstrophices (ollagen Rel Res 5:349) 35.4

9. Eve DR, (1)ten MP. Shapiro I). Wilkinson RII. Vawter 1986 . Vonexpression of cartilage type il collagen in a case of I anger-Saksino achondrogenesis. Am J Hum (icne1.39:52-67

10. (ay. S fine JI) 1986 lmmunization, isolation, and characteriation of pols and monoclonal antibodies apanst collagen for use in immuno histochemistrv. Methods !nzymol (in press)

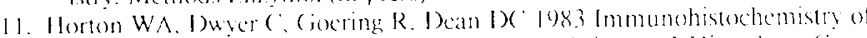
types I and II collagen in undecalcified skeletal tissues. I Histechem (yte- chem $31: 417-425$

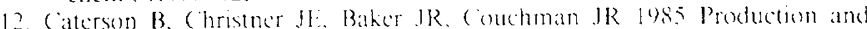
characterization of monedenal antibedies directed agatinst connective tissue proteoglycans. Fed Proc 4t:0106-011:3

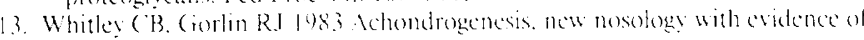

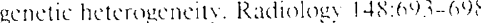

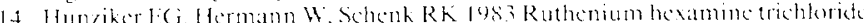
(RHIT)-mediated interaction between plasmalemmal components and pericollular matrix proteogleans is responsible for the preservation of chondrocytic plasma membranes in situ during cartibage fixation. I f fistochem (vitochem $31: 717-727$

15. Hogan D). Smith (ilf 1982 I neonventional application of standard light and electron immunocytochemical analysis to aldehyde-tixed araldite-embedded tissues. J Histochem (ytochem 30$): 1301-1.306$

16. Hsu SM. Raine 1. fanger 11 lgs! lese of avidin-biotin-peroxidase comples (ABC) in immunoperoxidase techniques. J Histochem (ytochem 24:577. 580

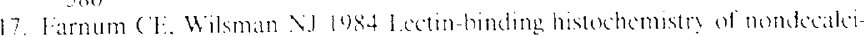
fied growth plate cartilage. I Histochem (ytochem .2.59, 3-6107

18. Timmerman $B$. Thies 11 198. Alterations of lectin binding dumb chondrogenesis of mousc limb huds. Histechemister $81: 35: 361$

19. Rimoin D) Hall \& Maroteaux P' 1979) International nomenclature of constitutional discalses of bonc. Birth Defects $15:(1) 0(0-000)$

20. Borochowitz 7. (Ornoy A. Lachman R. Rimoin D) 1986 Achondrogenesis.

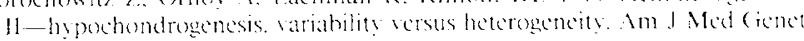
$24: 273-288$

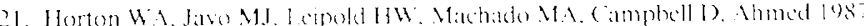

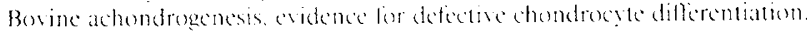
Bone $8: 191 \quad 197$

22. Moore KJ. 1977 the Iswetomg Human. WB Saunders. Philadelphia, pe $301-326$

23. Solursh $\mathrm{M} 198 \mathrm{~g}$ (cll and matrix interactions during limb chondrogenesis it vitro In. The Role of lextracellular Matrix in Bevelenment. Vlan R I iss. vew York. pp $277 \ldots 303$

24. Dessau $W$, won det Marh 11 , won der Mark $k$, Fisher $S 1980$ (hanges in the patterns of collagens and tibronectin during limb-bud chondrogenesis. timorvol Fy 1 lorphol $57.51-603$

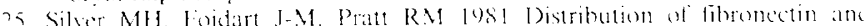
collagen during mouse limb and palate development. Deflerentiation $18: 1+1$ 14 )

26. Aulthouse 11 . Solursh 11 1980 The detection of a precartilage hlastem: specilic Slycoprotein. 1)o Bicol (in press)

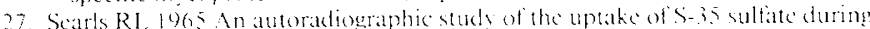
differentiation of limb bud cartilage. I) ex Biol $11: 155$ - 16.8

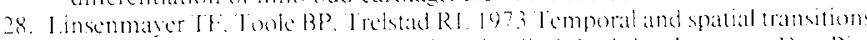
in collagen wpes during embronic shick limb bud development. I)es Biol $35: 232-230$

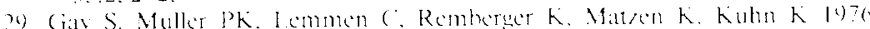
Immunobistological study on collagen in cartilage-bonc metamorphosis and

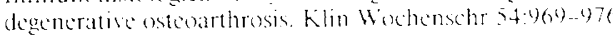

30. von der Mark $K 1980$ lmmunological studies on collagen type transition in chondrogenesis (ur lop B) B Biol 14:190_236

3 Dessiu $W$ Sasse J limpl R. Jilek J. won der Mark $k$ 1978 Sinthesis and extraccllular depessition of hibronectin in chondrocyte cultures. I (cill Biol $74: 342 \cdots 355$

32. Horton WA. Machado M: Mtterations in the cxtacellular matris during endochondral ossification in man. J Orthop Res (an press

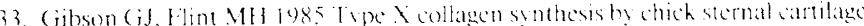

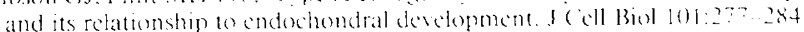

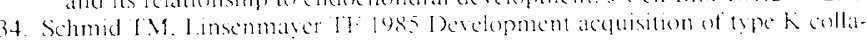
gen in the cmbryonic (hick tibiotarsus. Dow Biol $107: 37$, is 1

3: Benya PI) Vimn 11! 1979 The stabilits of the collagen phenotype during

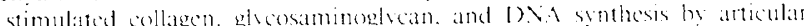
cartilage organ cultures Arch Biexhem Biophys 192: 227 - 53

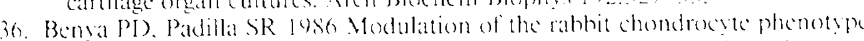
by retinoic acid terminates $1 y p e$ II collagen synthesis without inducing type l collagen, the modulated phenouse differs from that produced by subut ture J)e Biol $118: 29)(6-3) 5$

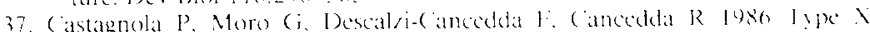
collagen sinthesis during in vitro dewelopment of chich embon tibial chondrocites. J ( ill Biol 102:2310-2317

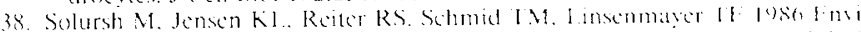

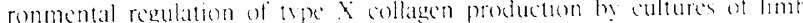

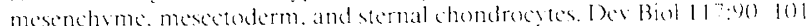

